\title{
Beam induced deposition of platinum using a helium ion microscope
}

\author{
Colin A. Sanford, ${ }^{\text {a) }}$ Lewis Stern, Louise Barriss, Lou Farkas, \\ Mark DiManna, and Russ Mello \\ Carl Zeiss SMT, Inc., One Corporation Way, Peabody, Massachusetts 01960 \\ Diederik J. Maas \\ TNO Science and Industry, Stieltjesweg 1, 2628 CK Delft, The Netherlands \\ Paul F. A. Alkemade \\ Kavli Institute of Nanoscience, Delft University of Technology, Lorentzweg 1, 2628 CJ Delft, \\ The Netherlands
}

(Received 7 July 2009; accepted 17 August 2009; published 2 December 2009)

\begin{abstract}
Helium ion microscopy is now a demonstrated practical technology that possesses the resolution and beam currents necessary to perform nanofabrication tasks, such as circuit edit applications. Due to helium's electrical properties and sample interaction characteristics relative to gallium, it is likely that the properties and deposition characteristics of beam induced deposited films will be different than those produced using gallium focused ion beam technology. However, there is at this date very little literature discussing the use of helium beams for beam induced chemistry or characterization of the resulting films. In this article, the authors present initial results regarding the deposition of platinum using a helium ion microscope and a gaseous organometallic precursor. Within this work a Carl Zeiss ORIONTM helium ion microscope was used along with an OmniGIS unit to deposit platinum while exploring a variety of controllable parameters such as beam current, beam overlap, and size of deposition. () 2009 American Vacuum Society. [DOI: 10.1116/1.3237095]
\end{abstract}

\section{INTRODUCTION}

Material modification via beam induced deposition and etching is an important nanofabrication technique. A specific nanofabrication application of great technical and monetary importance is that of circuit editing (CE). Circuit editing is a powerful technique for rapid debug and modification of integrated circuits. ${ }^{1}$ In recent years, the principal tool employed for circuit edit and device modification has been the gallium focused ion beam $\left(\mathrm{Ga}^{+} \mathrm{FIB}\right) \mathrm{Ga}^{+}$FIB can be used for both the deposition and removal of materials. The deposition process normally takes place by introducing gaseous precursors into the work chamber simultaneously with scanning the beam. ${ }^{2}$ The interaction of the beam, substrate, and precursor results in the deposition of a thin film. Both metals and insulators can be deposited using this method. As device nodes have become progressively smaller, $\mathrm{Ga}^{+}$FIB CE technologies have become increasingly difficult to utilize due to problems associated with the inherent beam tails of $\mathrm{Ga}^{+}$FIBs and the fact that gallium is electrically active in silicon devices. $\mathrm{Ga}^{+}$FIB poses other challenges as the circuit geometries continue to decrease, such as parasitic sputtering while imaging.

Helium ion beams are now available in the form of high resolution microscopes with beam parameters appropriate for circuit edit applications as well as general purpose nanofabrication. ${ }^{3}$ Due to the mass difference between helium and gallium, along with the different electrical properties of inert helium and electrically active gallium, it is reasonable to think that helium ion beams, or beams, based on other

\footnotetext{
a)Electronic mail: c.sanford@smt.zeiss.com
}

inert species, may provide a useful alternative to $\mathrm{Ga}^{+} \mathrm{FIB}$ for $\mathrm{CE}$ and other nanofabrication applications. In this article, we present initial results of platinum depositions using a helium ion beam provided by the ORIONTM helium ion microscope (HIM).

\section{BACKGROUND}

Beam induced deposition is a delicate balance of competing physical and chemical kinetics. A detailed description of the competing dynamics can be found in the recent review article by Utke et al. ${ }^{4}$ Deposition of a material is the result of the interaction between the incident beam, the substrate, and the chemical precursor which is allowed to enter the system. The precursor typically enters the system in close proximity to the beam through a delivery needle such that the local pressure is relatively high where the beam impinges on the substrate, but the overall average pressure within the sample chamber remains relatively unchanged. Several different interactions are possible. The primary beam may directly interact with precursor molecules on the surface of the substrate, dissociating them and allowing the constituents to undergo chemical reactions with the substrate. Alternatively, the adsorbed precursor molecules may be dissociated by the energetic particles produced by the incident beam, such as backscattered and secondary electrons, or displaced atoms. The heat energy created by the incident beam within the target substrate may also play a role in dissociating the adsorbed precursor molecules. ${ }^{5}$ Whatever the mechanism of dissociation and subsequent material deposition, the rate of reaction must be greater than the rate of parasitic material removal due to sputtering by the primary beam of the surface. 
TABLE I. Value assigned to fixed experimental parameters.

\begin{tabular}{ll}
\hline \hline Parameter & Fixed value \\
\hline $\begin{array}{l}\text { Exposure dose } \\
\text { Beam energy }\end{array}$ & $2 \mathrm{nC} / \mu \mathrm{m}^{2}$ \\
$\begin{array}{l}\text { Precursor } \\
\text { temperature }\end{array}$ & $30 \mathrm{kV}$ \\
$\begin{array}{l}\text { Substrate bias } \\
\text { Substrate }\end{array}$ & $30^{\circ} \mathrm{C}$ \\
& $0 \mathrm{~V}$ \\
Injector angle & $\mathrm{Cr}$ on glass mask \\
Injector height above & blank \\
substrate & $30^{\circ}$ \\
\hline
\end{tabular}

It is well known ${ }^{2,4}$ that many parameters affect the rate of material deposition or removal when using precursor gases to enable the deposition or enhance the etch rate of a material. Among the extensive parameters listed are beam pixel dwell time, beam current, beam species, beam pixel spacing, precursor pressure, beam energy, and sample bias. Depending on the application, the desired physical properties of the deposited film vary. The application of circuit edit is one of the more demanding applications of beam induced materials deposition. Critical parameters in this case include electrical conductivity, resistivity, and deposition rate. Deposition rate is typically measured in $\mu \mathrm{m}^{3} / \mathrm{nC}$.

The experimental equipment used for this initial investigation of platinum deposition by a helium ion beam consisted of an ORIONTM HIM and an OmniGIS (Ref. 6) gas injection system (GIS). (Methylcyclopentadientyl)trimethyl platinum $\left(\mathrm{C}_{9} \mathrm{H}_{16} \mathrm{Pt}\right)$ was used as the gaseous precursor for the deposition of platinum. After completing several screening experiments, it was decided to more thoroughly investigate the impact of three parameters upon the deposition rate and at. $\%$ composition within platinum deposits. The factors investigated were beam current, pixel spacing, and overall deposit size.

\section{EXPERIMENTAL TECHNIQUE AND ANALYSIS}

Statistically design of experiments (DOEs) provide a powerful means for determining the statistical significance of experimental factors and are ideally suited for investigating multivariable problems in which interactions are expected to occur between the experimental factors. ${ }^{7}$ In this initial experiment, a full factorial statistically designed experiment was used. The experimental design was a 3 factor, 2 level full factorial design with a full replicate and center points. The JMP software package was used for analyzing the resulting data. The center point of the experimental parameters was empirically selected based on several screening experiments conducted in advance. The center point of the experimental parameters was chosen to be

(1) beam current $=3.5 \mathrm{pA}$,

(2) deposit size $=625 \times 625 \mathrm{~nm}^{2}$, and
TABLE II. DOE summary table presenting the parameter configuration and results of each experimental run.

\begin{tabular}{cccccc}
\hline \hline Run No. & $\begin{array}{c}\text { Pixel } \\
\text { spacing } \\
(\mathrm{nm})\end{array}$ & $\begin{array}{c}\text { Beam } \\
\text { current } \\
(\mathrm{pA})\end{array}$ & $\begin{array}{c}\text { Box size } \\
(\mathrm{nm})\end{array}$ & At. \% Pt & $\begin{array}{c}\text { Depo } \\
\text { rate } \\
\left(\mu \mathrm{m}^{3} / \mathrm{nC}\right)\end{array}$ \\
\hline 1 & 2 & 3.5 & 625 & 16.90 & 0.34 \\
2 & 1 & 6 & 1000 & 19.50 & 0.33 \\
3 & 2 & 3.5 & 625 & 15.60 & 0.34 \\
4 & 1 & 6 & 1000 & 19.90 & 0.28 \\
5 & 1 & 6 & 250 & 14.60 & 0.30 \\
6 & 3 & 6 & 1000 & 15.00 & 0.25 \\
7 & 2 & 3.5 & 625 & 13.90 & 0.31 \\
8 & 1 & 1 & 1000 & 10.90 & 0.49 \\
9 & 1 & 1 & 250 & 11.90 & 0.54 \\
10 & 2 & 3.5 & 325 & 14.30 & 0.25 \\
11 & 3 & 6 & 250 & 13.86 & 0.24 \\
12 & 1 & 1 & 250 & 11.50 & 0.60 \\
13 & 3 & 6 & 250 & 13.90 & 0.26 \\
14 & 3 & 1 & 1000 & 8.40 & 0.43 \\
15 & 1 & 6 & 250 & 15.20 & 0.30 \\
16 & 3 & 1 & 1000 & 9.14 & 0.44 \\
17 & 3 & 6 & 1000 & 15.30 & 0.23 \\
18 & 3 & 1 & 250 & 11.30 & 0.54 \\
19 & 3 & 1 & 250 & 11.60 & 0.45 \\
20 & 1 & 1 & 1000 & 10.90 & 0.34 \\
\hline \hline
\end{tabular}

(3) pixel spacing $=2 \mathrm{~nm}$.

All other known variables were held fixed. The values of the fixed parameters are presented in Table I. All platinum depositions of the DOE were accomplished during a single work session. Upon completion of the depositions, the amount of material was analyzed by imaging each deposit using an atomic force microscope (AFM). The computed volume of deposited material was then divided by the total dose of the exposure to obtain the deposition rate in $\mu \mathrm{m}^{3} / \mathrm{nC}$. A summary table of the DOE is presented in Table II. The at. \% of platinum was determined by using energy dispersive spectroscopy. Figure 1 presents a secondary electron image obtained of the deposit array corresponding to the DOE summary table. Figure 2 presents a typical AFM image of a single deposit.

Once the deposition rate and at. \% for each experimental run had been determined, the data were statistically analyzed using the JMP statistical analysis package. A model was fit to the data, including all interaction terms up to degree 2. The effect of an experimental factor in a model was tested for significance by comparing the sum of squared residuals to the sum of squared residuals of the model with the effect of the experimental factor removed. Residual errors that are much smaller when the effect is included in the model confirm that the effect is a significant contribution to the fit of the model. The graphical display of an effect's significance test is called a leverage plot. This kind of plot shows for each point what the residual would be both with and without that effect in the model. Effect Leverage plots were used to determine the relative importance of the various controlled parameters. After determining which experimental parameters 


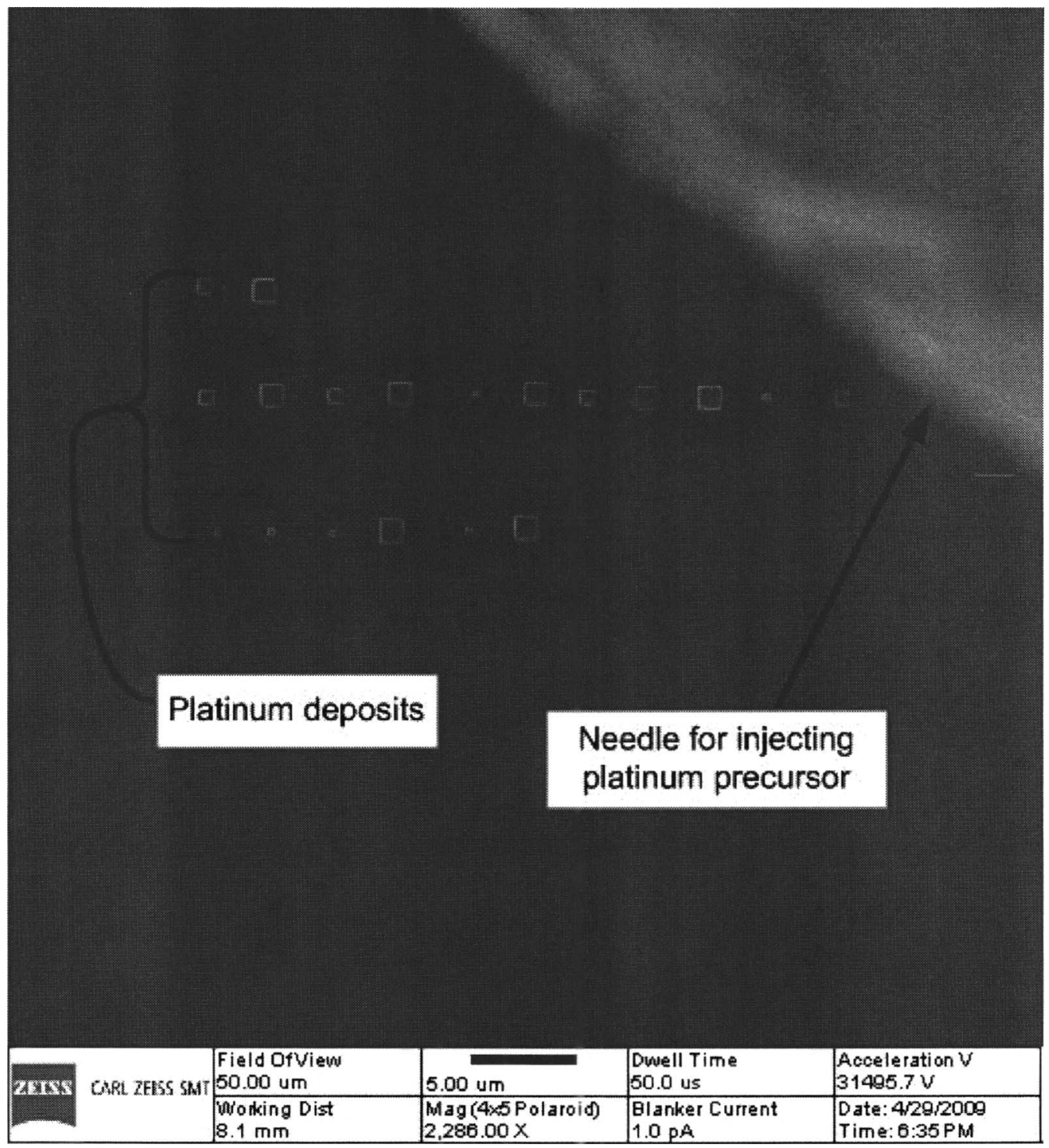

FIG. 1. Secondary electron image of platinum deposits corresponding to the DOE summary table. The GIS needle is visible in the upper right hand corner of the image.

were statistically significant, a mathematical model was created to model the data. The resulting prediction formula inclusive of all primary factors and second order cross terms for the at. \% platinum was determined to be

$$
\begin{aligned}
\text { At. } \%= & 13.68+0.99[\mathrm{PS}(\mathrm{nm})-2] \\
& +2.60\left[\frac{\mathrm{BC}(\mathrm{pA})-3.5}{2.5}\right] \\
& +0.32\left[\frac{\mathrm{BS}(\mathrm{nm})-625}{375}\right]-0.40(\mathrm{PS}(\mathrm{nm})-2) \\
& \times\left[\frac{\mathrm{BC}(\mathrm{pA})-3.5}{2.5}\right]-0.67(\mathrm{PS}(\mathrm{nm})-2) \\
& \times\left[\frac{\mathrm{BS}(\mathrm{nm})-625}{375}\right]+1.19\left[\frac{\mathrm{BC}(\mathrm{pA})-3.5}{2.5}\right]
\end{aligned}
$$

$$
\left.\times \frac{\mathrm{BS}(\mathrm{nm})-625}{375}\right]
$$

where PS is the pixel spacing, $\mathrm{BC}$ is the incident beam current, and BS is the deposited box size. Figure 3 shows how well the derived mathematical model for predicting at. \% platinum fits to the experimental data.

Examination of all leverage plots associated with the at. \% platinum deposition indicated that the box size parameter was not a statistically significant factor in determining the percent platinum within the deposits, when viewed in isolation. However, the leverage plot for the interaction between beam current and box size did show statistical significance, and thus all factors including interaction terms were used to construct the analytical predictive model for percent platinum concentration in the depositions. The leverage plots 


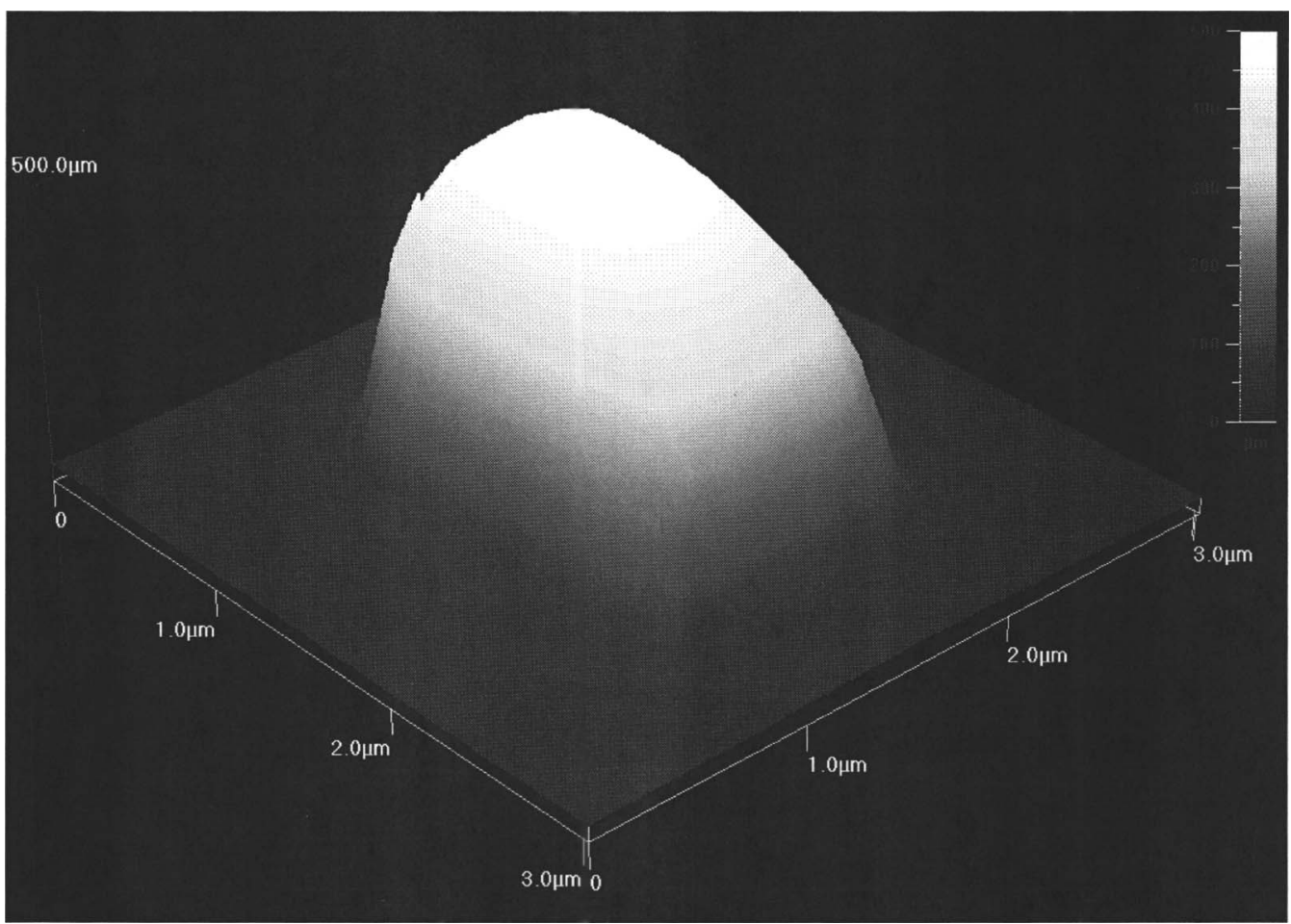

FIG. 2. AFM image of the platinum deposit.

for beam current, box size, and their interaction are shown in Fig. 4. Statistical significance is generally accepted as being real when both the regression line and the $95 \%$ confidence bands for a parameter cross the horizontal mean. This is the case for the beam current parameter, but not for the box size.
However, when the interaction between box size and beam current is examined, we see that the combined interaction term is significant. This is further demonstrated by examining the accuracy of the prediction model when the interaction term is eliminated. When the beam current and box size are

\section{Measured vs predicted atomic \% platinum}

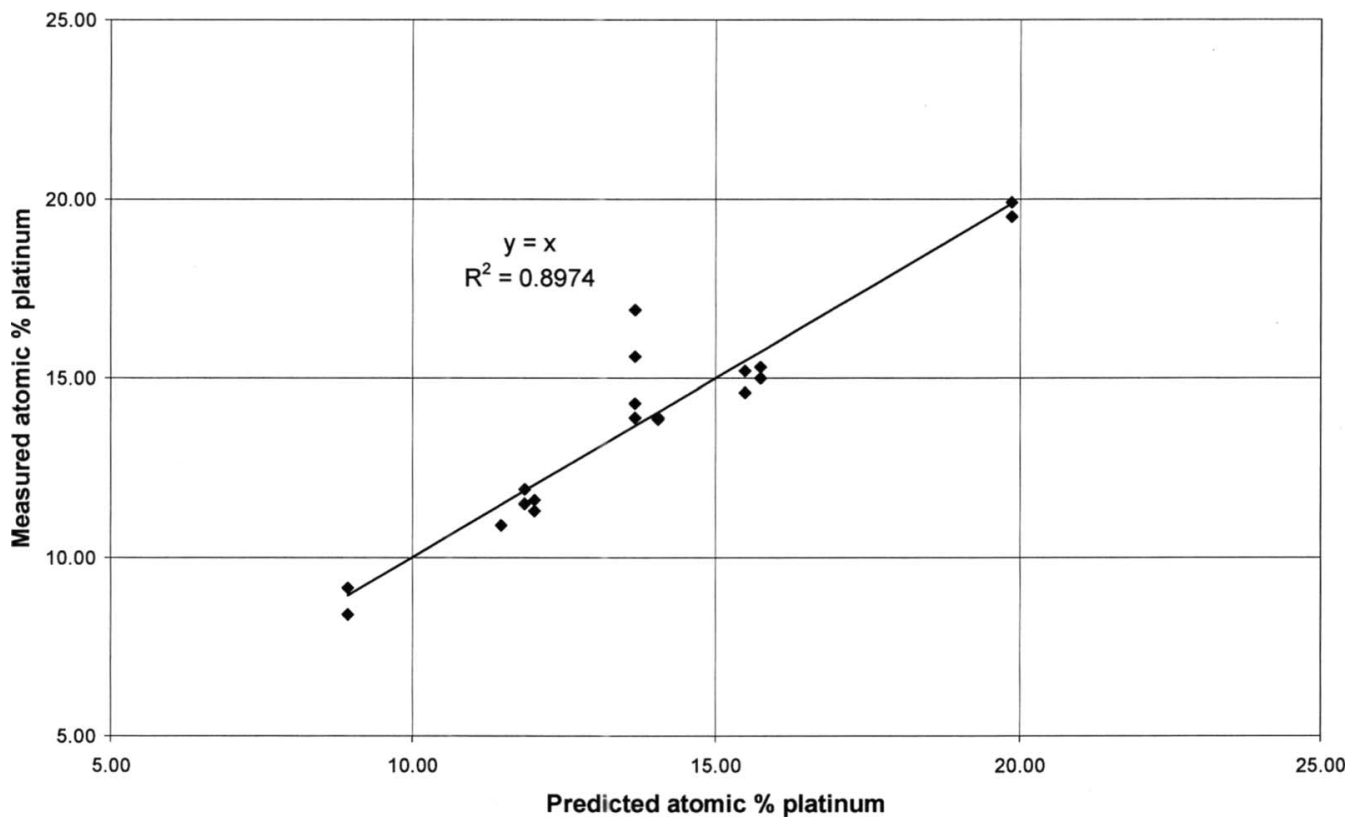

FIG. 3. Graph of measured vs predicted at. \% platinum in deposited films. The predicted value is based on the mathematical model constructed from the DOE results. The relatively high $R^{2}$ value indicates that the model explains all observed variations to a high degree. 


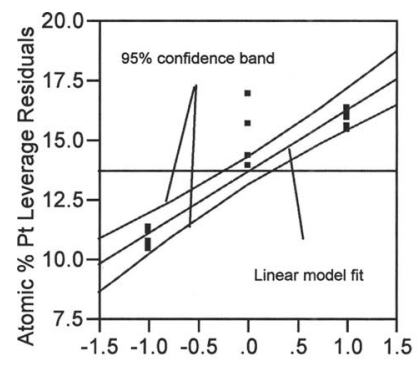

a) Beam current (level setting)
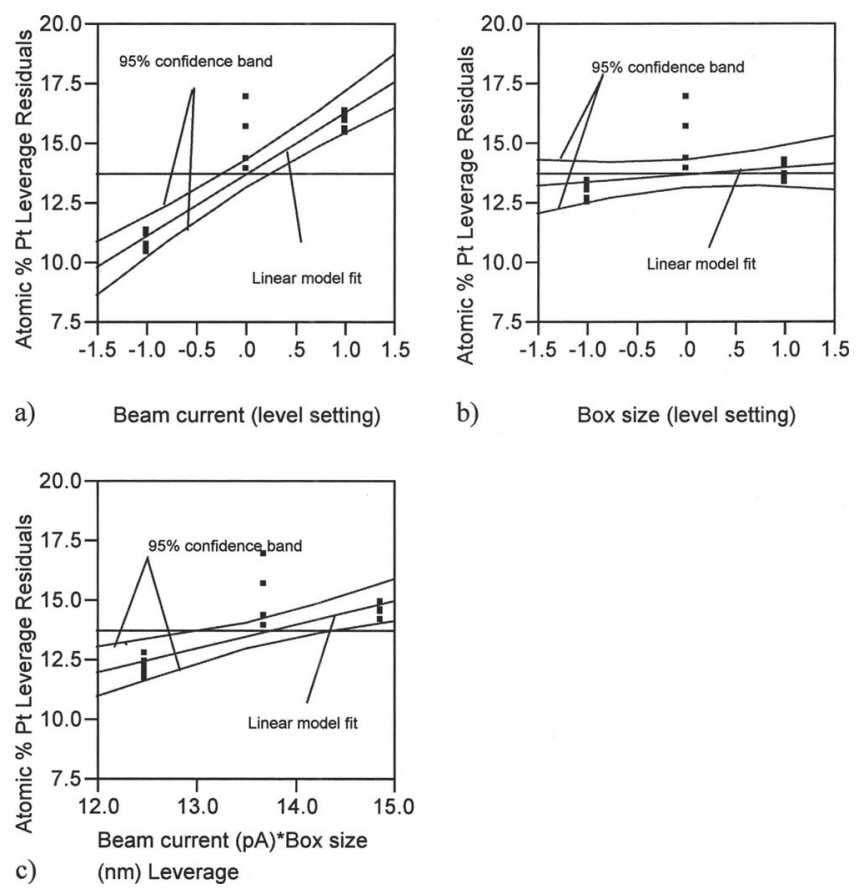

FIG. 4. Leverage plots showing best fit along with $95 \%$ confidence bands. (a) Leverage plot for beam current showing strong statistical significance. (b) Leverage plot for box size. The fact that the confidence bands do not cross the horizontal mean response indicates a lack of statistical significance of the box size parameter. (c) Leverage plot for the interaction of box size and beam current showing that the interaction term is statistically significant. kept as primary factors, but the interaction term is dropped from the model, the $R^{2}$ value for the fitting function drops from 0.89 to 0.76 , as shown by Fig. 5 .

In a similar fashion to the technique used to derive an analytical model for at. \% concentration of platinum, a model for describing the deposition rate was created. For the experimental conditions used, it was found upon examination of the leverage plots that pixel spacing had no statistically significant impact upon the deposition rate. Only the beam current and box size were important. The interaction between the two was also shown to have some significance. The resulting analytical predictive model for deposition rate was determined to be

$$
\begin{aligned}
\operatorname{Deporate}\left(\mu \mathrm{m}^{3} / \mathrm{nC}\right)= & 0.363-0.11\left[\frac{\mathrm{BC}(\mathrm{pA})-3.5}{2.5}\right] \\
& -0.03\left[\frac{\mathrm{BS}(\mathrm{nm})-625}{375}\right] \\
& +0.026\left[\frac{\mathrm{BC}(\mathrm{pA})-3.5}{2.5}\right] \\
& \times\left[\frac{\mathrm{BS}(\mathrm{nm})-625}{375}\right]
\end{aligned}
$$

where BC is the incident beam current and BS is the deposited box size. Figure 6 shows how well the derived mathematical model for deposition rate matches the observed deposition rates.

A generally accepted model $^{2}$ for ion beam induced deposition describes three regimes of beam current density. In the first regime, the beam current is low such that there is insufficient beam current to decompose all of the available pre-

\section{Measured vs predicted atomic \% platinum}

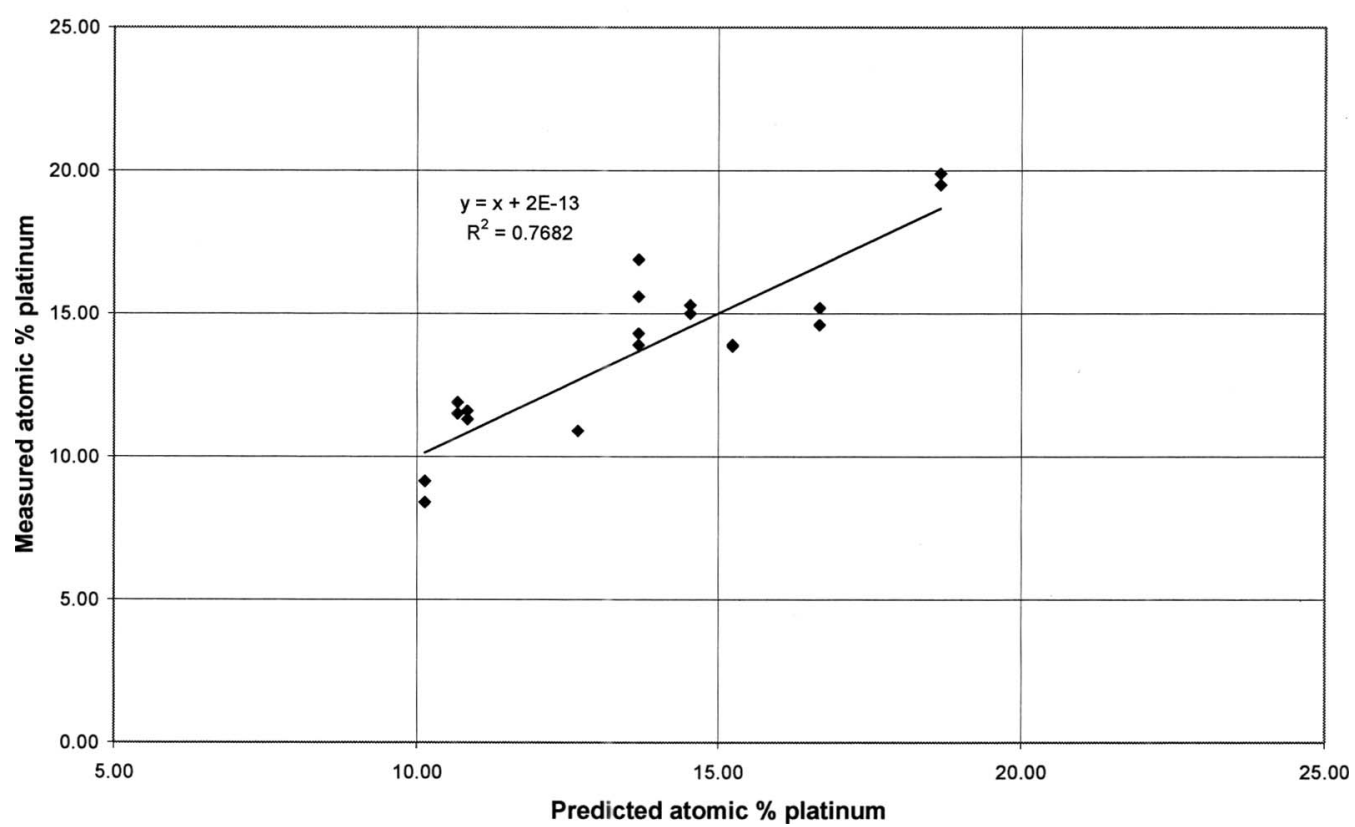

Fig. 5. Measured vs predicted at. \% platinum in deposited films when the interaction between box size and beam current is eliminated from the model. 
Measured vs predicted deposition rate $\left(\mu \mathrm{m}^{3} / \mathrm{nC}\right)$

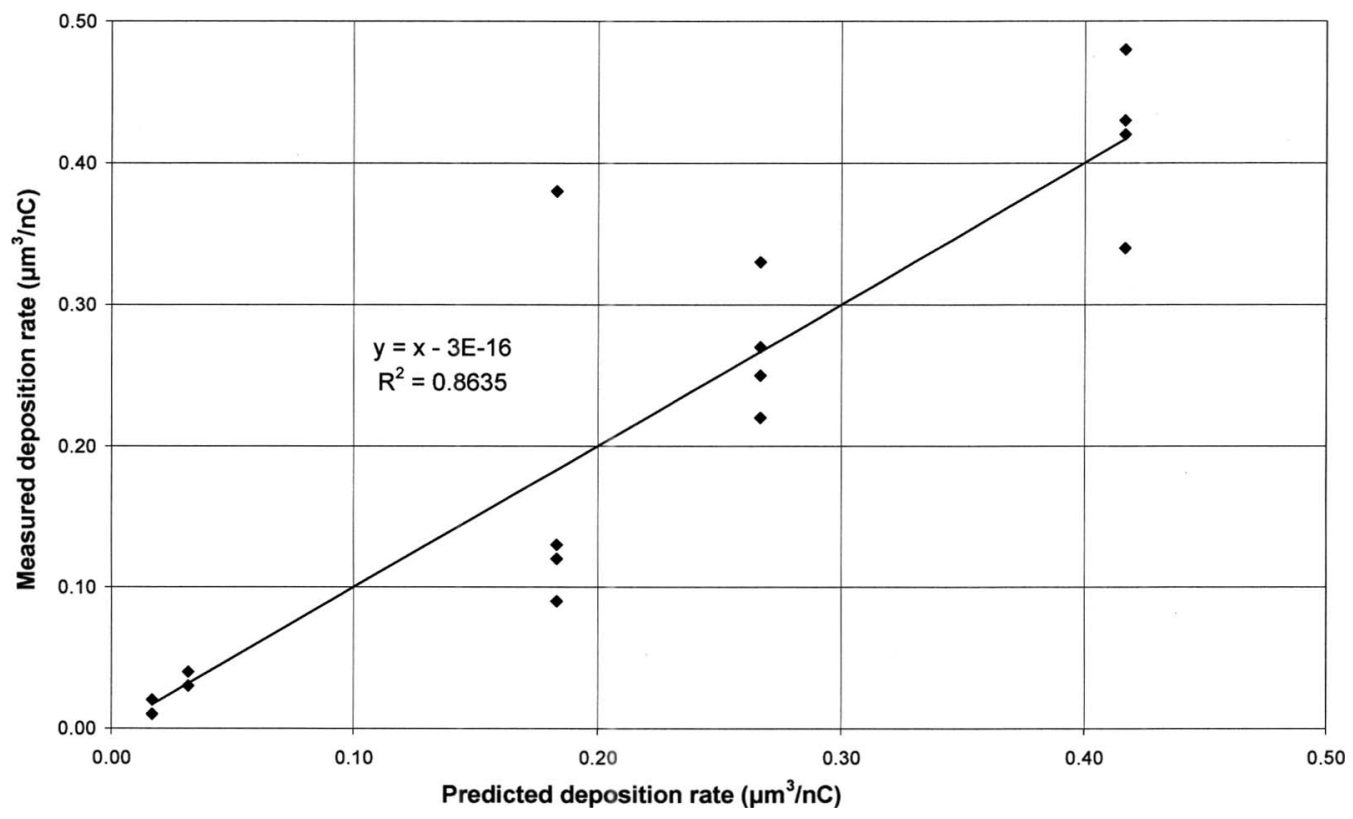

FIG. 6. Measured vs predicted deposition rate of platinum.

cursor adsorbed onto the surface. In this regime the deposition rate is low. The second regime occurs when the beam current is sufficient that almost all of the precursor gases are decomposed during each pixel dwell period of the beam. As the ion current is further increased, the precursor gas is fully decomposed at each pixel dwell in a time frame that is short compared to the pixel dwell time. In this regime the deposition decreases from a maximum value obtained in regime 2 to zero, beyond which point the sputter rate of the ion beam dominates the deposition rate. Using $\mathrm{Ga}^{+} \mathrm{FIB}$ the accepted beam current range for regime 2 is $2-6 \mathrm{pA} / \mu \mathrm{m}^{2}$ when depositing platinum. ${ }^{8}$ This current density refers to the pattern current density and is defined as the probe current divided by the pattern area. Within this experimental work, there was not a strong correlation found between deposition rate and pattern current density. This may be because the effect is dominated by primary beam current density and not the pattern current density. It is reasonable to assume that the primary beam current density plays an important role as well as the pattern current density. It is noted here that the local current density is approximately $10^{6}$ times greater than the pattern current density. Due to the superior current density profile of helium ion beams relative to $\mathrm{Ga}^{+}$FIBs, which is largely due to superior $\delta E$ of the helium beam, it is believed that the local current density of the helium beam is greater than that of a typical $\mathrm{Ga}^{+}$FIB. The high local current density of the HIM may cause the boundaries between the three deposition regimes to differ significantly from the values typically seen with $\mathrm{Ga}^{+}$FIB. Although a statistically significant correlation between pattern current density and deposition rate was not observed, a correlation between primary beam current and deposition rate was observed, as shown in Fig. 7. The fact that the deposition rate decreases with in- creasing beam current indicates that the depositions likely suffer from depletion of the gaseous precursor during the process.

\section{DISCUSSION}

Using the derived analytical model above for at. \% of platinum, the appropriate parameters needed to optimize the percent platinum concentration were determined. The appropriate parameter values were determined to be a pixel space of $1 \mathrm{~nm}$, box size of $1500 \mathrm{~nm}$, and beam current of $15 \mathrm{pA}$. Several additional deposits were made using the new parameters but the best obtained value remained nearly $20 \%$, whereas a value of $\sim 40 \%$ was expected based on the model. The discrepancy is believed to be due to the effect of an unidentified critical parameter that had not been controlled. The additional deposits were created on a different day and with a different tool operator. It is known that the new depositions were created with a beam energy of $20 \mathrm{kV}$ instead of the $30 \mathrm{kV}$ used in the original DOE. Differences in the beam interaction at the two different energies, such as differences in secondary electron yield, or heat generation may explain the discrepancy. It has been reported that for the case of electron beam deposition of platinum the deposition rate is a function of primary beam energy, and the dependence of secondary electron yield as a function of primary beam energy is believed to be responsible. ${ }^{9}$ An alternative explanation is that the local precursor pressure may have been different on the different days. As described earlier, the initial deposits appear to have been created in a "gas depleted" regime of the parameter space, and hence any changes from day to day of the local pressure would likely have a strong influence upon the observed deposition rate. Despite the dis- 
Measured deposition rate vs beam current

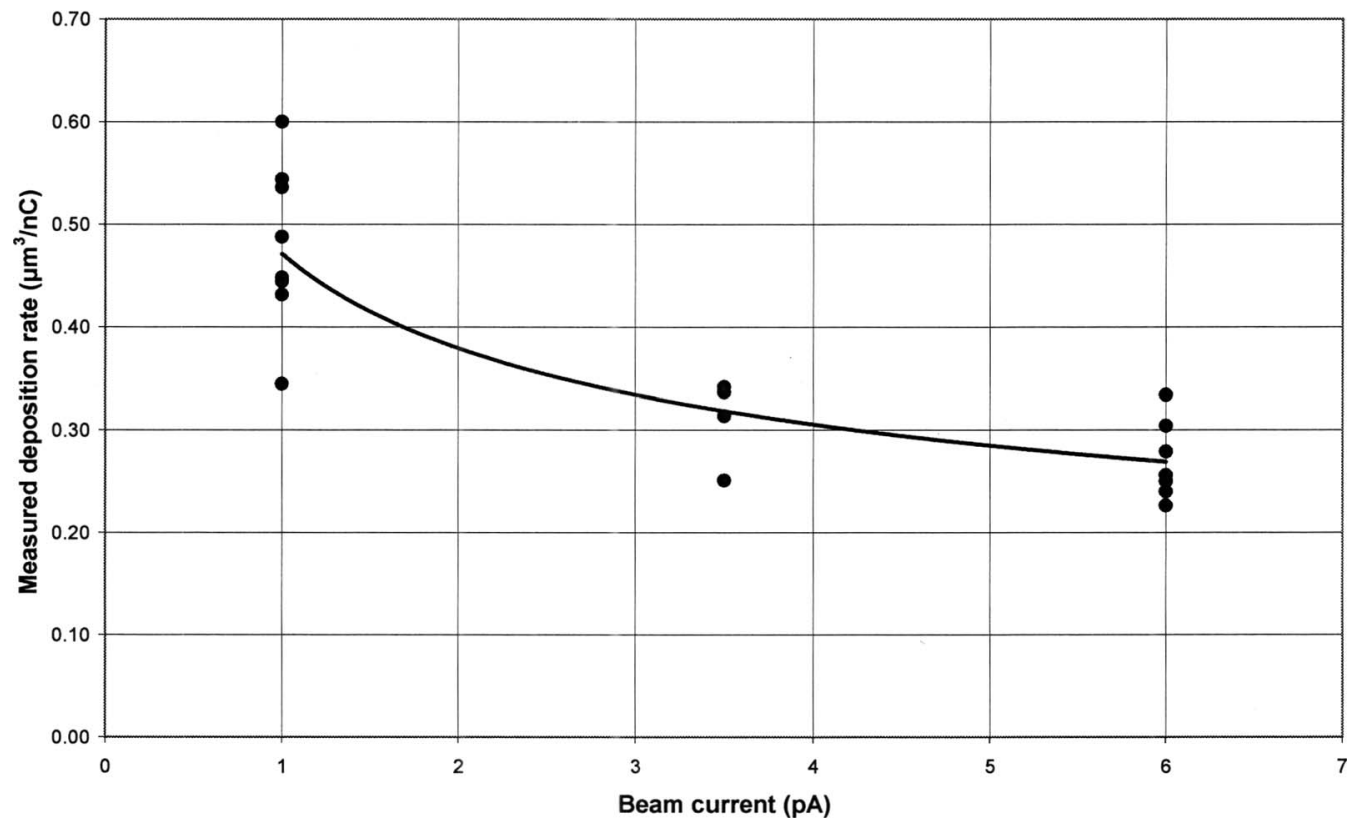

FIG. 7. Measured deposition rate vs sample current. The decreasing deposition rate with increasing sample current is indicative of running in a gas depletion regime.

crepancy between the predicted and the measured results of the additional platinum deposits, it is believed based on the goodness and linearity of fit between the model and the experimental data obtained from the DOE that obtainable percentages of platinum can be easily increased if all parameters are controlled. Further investigation is required.

Comparison of the at. $\%$ platinum contained in the initial depositions using a helium ion beam with the values in lit- erature for electron beam and $\mathrm{Ga}^{+}$FIB deposited films suggests that the initial experimental film quality obtained in the DOE is similar to that obtained with e-beam. Electron beam induced platinum depositions are reported to have 15-25 at. $\%$ of platinum. ${ }^{9,10}$ Platinum depositions created by $\mathrm{Ga}^{+}$ FIB processes typically provide higher concentrations of platinum. The reported at. \% concentrations range from 25 to 45 . $^{11,12}$ The values of the observed deposition rate of plati-

Atomic \% platinum vs measured deposition rate $\left(\mathrm{um}^{3} / \mathrm{nC}\right)$

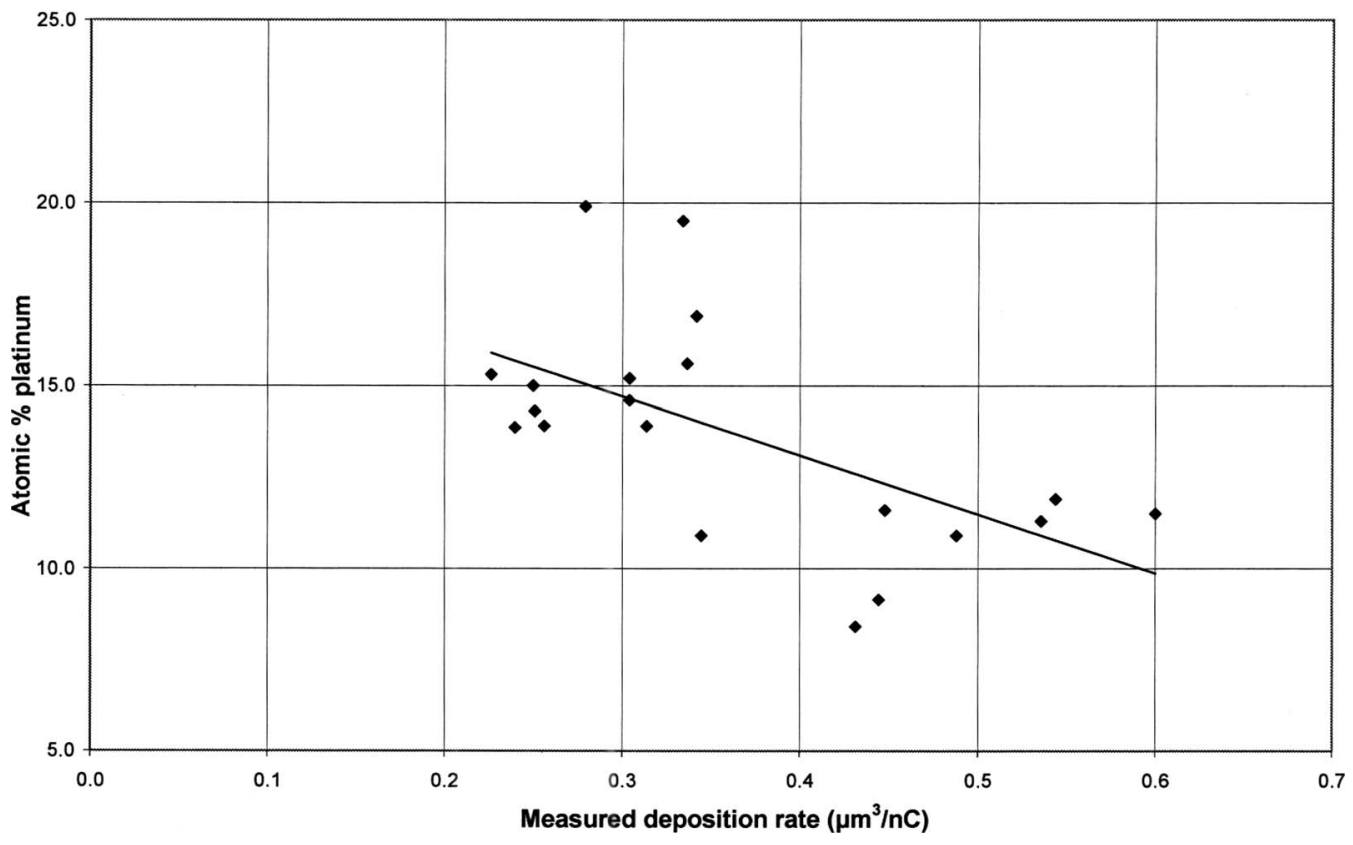

FIG. 8. Experimental platinum concentration vs the observed deposition rate. 
num are similar to that reported in literature for $\mathrm{Ga}^{+}$FIB depositions. Reported platinum deposition rates for $\mathrm{Ga}^{+}$FIB are $\sim 0.2-0.5 \mu \mathrm{m}^{3} / \mathrm{nC}^{8}$. This yield is quite favorable when contrasted with the relatively low deposition rate of $<0.01 \mu \mathrm{m}^{3} / \mathrm{nC}$ for electron beam induced platinum deposits. ${ }^{9}$ The relationship between the observed deposition rate and at. \% platinum is illustrated in Fig. 8. The data contain considerable scatter, but generally indicate an inverse relationship between the two parameters. The at. \% of platinum decreases as the deposition rate increases. This is consistent with reported results ${ }^{11}$ for $\mathrm{Ga}^{+}$FIB depositions of platinum in which the measured resistivity of the deposits increased as the deposition yield increased.

In addition to depositing metals, any practical CE tool must be capable of also etching materials. At this date only preliminary experiments regarding etching using $\mathrm{XeF}_{2}$ have been performed, and more work is needed to quantify results. However, since physical sputtering is often a key component of any beam induced etching process, it is reasonable to expect that the results obtained from a helium based CE tool with regard to material removal will be somewhere between those obtained with e-beam and $\mathrm{Ga}^{+}$FIB tools due to the mass of the helium ion. Helium may prove advantageous, however, because it provides not only a sputtering component not available with electrons but also an ability to obtain very high resolution images at low ion flux that can be used for the purposes of nondestructive endpoint detection in the absence of gases, unlike $\mathrm{Ga}^{+}$which continues to physically mill the sample.

\section{CONCLUSIONS}

The effects of beam current, box size, and pixel spacing on helium induced platinum depositions were studied by using a full factorial design experiment. Over the range of parameters studied in the DOE, it was found that platinum deposits could be obtained with at. \% compositions ranging from approximately 8 to 20 . These values are consistent with that obtained from electron beam induced depositions. A prediction model was created based on the results of the DOE. Based on the prediction model, it was expected that $40 \% \mathrm{Pt}$ would be achievable by adjustment of the three controlled parameters, but this was not achieved. The reason for this is not known and is currently under investigation. However, at this point in time only a small fraction of the complete parameter space that affects the concentration of platinum has been explored. Traditional variables, such as dwell time have not been explored, and there is significant optimization work to be completed. The DOE also indicated that interactions between the controlled variables are important. The observed deposition rates for the platinum films varied between 0.23 and $0.60 \mu \mathrm{m}^{3} / \mathrm{nC}$, consistent with the results obtained for $\mathrm{Ga}^{+}$FIB depositions. The data indicate that the deposits were done in a gas depletion regime of the process parameter space. These early results are encouraging. Given the relatively short amount of time that has been available for studying the properties of HIM induced depositions, and the very large parameter space to be explored, along with the fact that the early results are comparable to those reported in literature for $\mathrm{Ga}^{+} \mathrm{FIB}$, it seems reasonable that continued optimization of deposition processes can lead to film quality that is superior to that provided by $\mathrm{Ga}^{+}$FIB.

${ }^{1}$ R. H. Livengood, P. Winer, and V. R. Rao, J. Vac. Sci. Technol. B 17, 40 (1999).

${ }^{2}$ N. Yao, Focused Ion Beam Systems (Cambridge University Press, Cambridge, 2007).

${ }^{3}$ B. Ward, J. A. Notte, and N. P. Economou, J. Vac. Sci. Technol. B 24, 2871 (2006).

${ }^{4}$ I. Utke, P. Hoffmann, and J. Melngailis, J. Vac. Sci. Technol. B 26, 1197 (2008).

${ }^{5}$ J. Melengailis, P. C. Blauner, A. D. Dubner, J. S. Ro, T. Tao, and C. V. Thomson, Proceedings of International Symposium on Progress Physics and Modeling in Semiconductor Technology 1990 (unpublished), p. 652. ${ }^{6}$ OmniProbe, Inc., 10410 Miller Road, Dallas, Texas 75238.

${ }^{7}$ D. Montgomery, Design and Analysis of Experiments (Wiley, New York, 1991).

${ }^{8}$ Platinum Deposition Technical Note (FEI Company, Hillsboro, OR, 2003), PN 4035272 21851-B.

${ }^{9}$ S. Lipp, L. Frey, C. Lehrer, E. Demm, S. Pauthner, and H. Ryssel, Microelectron. Reliab. 36, 1779 (1996).

${ }^{10}$ A. Fernandez-Pacheco, J. M. De Teresa, R. Cordoba, and M. R. Ibarra, J. Phys. D: Appl. Phys. 42, 055005 (2009).

${ }^{11}$ J. Puretz and L. W. Swanson, J. Vac. Sci. Technol. B 10, 2695 (1992).

${ }^{12}$ T. Tao, J. Ro, J. Melngailis, Z. Xue, and H. Kaesz, J. Vac. Sci. Technol. B 8, 1826 (1990). 